\title{
Article \\ Reliability of Supply and the Impact of Weather Exposure and Protection System Failures ${ }^{+}$
}

\author{
Erlend Sandø Kiel ${ }^{1, *(D)}$ and Gerd Hovin Kjølle ${ }^{2}$ (D)
}

check for

updates

Citation: Kiel, E.S.; Kjølle, G.H. Reliability of Supply and the Impact of Weather Exposure and Protection System Failures. Appl. Sci. 2021, 11, 182. https://dx.doi.org/10.3390/ app11010182

Received: 20 November 2020 Accepted: 23 December 2020 Published: 27 December 2020

Publisher's Note: MDPI stays neutral with regard to jurisdictional claims in published maps and institutional affiliations.

Copyright: () 2021 by the authors. Licensee MDPI, Basel, Switzerland. This article is an open access article distributed under the terms and conditions of the Creative Commons Attribution (CC BY) license (https: / / creativecommons.org/ licenses/by/4.0/).
1 Department of Electric Power Engineering, Faculty of Information Technology and Electrical Engineering, Norwegian University of Science and Technology (NTNU), 7491 Trondheim, Norway

2 SINTEF Energy Research, 7034 Trondheim, Norway; gerd.kjolle@sintef.no

* Correspondence: erlend.kiel@ntnu.no

$+\quad$ This paper is an extended version of our paper published in 2019 IEEE International Conference on Environment and Electrical Engineering and 2019 IEEE Industrial and Commercial Power Systems Europe (EEEIC/I\&CPS Europe), Genova, Italy, 13 June 2019.

\begin{abstract}
Extreme weather is known to cause failure bunching in electrical transmission systems. However, protection systems can also contribute to the worsening of the system state through various failure modes-spontaneous, missing or unwanted operation. The latter two types of failures only occur when an initial failure has happened, and thus are more likely to happen when the probability of failure of transmission lines is high, such as in an extreme weather scenario. This causes an exacerbation of failure bunching effects, increasing the risk of blackouts, or High Impact Low Probability (HILP) events. This paper describes a method to model transmission line failure rates, considering both protection system reliability and extreme weather exposure. A case study is presented using the IEEE 24 bus Reliability Test System (RTS) test system. The case study, using both an approximate method as well as a time-series approach to calculate reliability indices, demonstrates both a compact generalization of including protection system failures in reliability analysis, as well as the interaction between weather exposure and protection system failures and its impact on power system reliability indices. The results show that the inclusion of protection system failures can have a large impact on the estimated occurrence of higher order contingencies for adjacent lines, especially for lines with correlated weather exposure.
\end{abstract}

Keywords: protection systems; failure bunching; extreme weather; reliability; HILP; extraordinary events

\section{Introduction}

Society is dependent on a reliable supply of electricity for its normal operation, and thus power outages can have severe consequences. Major blackouts are often due to multiple component outages, caused by environmental factors [1-5], and/or dependent failures such as protection system failures [6]. The increased probability of failure of one or more components in a short period of time due to extreme weather has been termed failure bunching, and models have been developed to capture such effects in power system reliability studies by using both analytical methods and Monte Carlo simulation methods [7-10]. Large blackouts are often a consequence of complex series of events, such as cascading failures [11]. A distinction in the structure of cascading blackouts is made in [12] between a triggering event, which can be simple component failure(s), and generation of propagating events, caused by preceding events and a change in the power system state.

One of the causes of propagating events is the misoperation of protection systems $[4,11,13]$. Overlapping outages of highly reliable components, whose probability is the product of the individual component outage probabilities, can be rather small even if their individual probabilities are relatively high. Dependent failures, such as protection system failures, can however lead to the same set of simultaneous component outages with a much higher probability than 
what the simultaneous independent outages might suggest [14]. This will in turn increase the risk of the scenario, as a function of its probability and consequence [15]. Previous studies have shown how protection system failures can have a significant impact on system reliability evaluation [16,17]. Combining the effects of failure bunching with protection system failures may give more realistic picture of the power system reliability assessment.

The hypothesis in this paper is that the combination of failure bunching effects and protection system failures will increase the risk of blackout events. To the best of our knowledge, this hypothesis was not investigated before in a quantifiable manner with respect to system reliability. The goal of this paper is to carry out investigations to verify the proposed hypothesis. The unique contributions made in this paper are as follows: A generalized compact system of equations using graph theory has been formulated to quantify the impact of protection system failures on power system reliability, based on [17]. Graph theory helps establish a systematic identification of propagation of transmission line failures due to misoperation of protection systems. These equations are then used to incorporate the effects of failure bunching due to harsh weather on the system reliability. Subsequently, these equations are applied to time-series of failure probability of transmission lines specifically due to wind conditions. It should be noted that the procedure is not only applicable to wind-dependent threats but can be used with any time-varying threats, e.g., due to other weather conditions.

The paper is structured as follows: In Section 2, previous work on failure bunching effects and protection system reliability is presented, together with a short review of graph theory and its use in this study of power systems reliability. In Section 3, a general and compact method for calculating reliability indices including protection system misoperations is presented, using both approximate methods and a time-series method. Section 4 presents a case study where the method is applied, before the paper is concluded in Section 5 . This paper is an extension of the work presented in [18], incorporating an extended literature review, updated equations, calculation of unavailability for the time-series method, additional illustrations as well as an extended case study and results.

\section{Failure Bunching and Protection System Failures}

Harsh weather has long been known to cause common cause failures within short periods of time, often termed failure bunching. One way of incorporating such effects is to use multi-state Markov models or similar approximate methods when calculating reliability, another is to utilize Monte Carlo simulation techniques [3,19,20]. In [19], the effect of failure bunching due to wind exposure is captured using historical failure data and a Bayesian updating scheme to estimate annual wind dependent failure rates of transmission lines. The annual failure rate is then spread out in time by combining fragility curve modeling and a dataset of historical wind speeds for the lines in question. This results in hourly time-series of wind dependent failure probability for the lines. This approach has been applied to create similar time-series of failure probability due to other weather effects such as icing and lightning, which is further used together with a Monte Carlo-based tool to calculate system consequences in terms of interrupted power and interruption costs in [21].

In [10] an analytical technique is used to calculate time-series of expected unavailability of transmission lines due to wind conditions, instead of using a Monte Carlo approach. For each hour, the probability of failure of the transmission line is paired with a distribution of outage duration. An iterative algorithm then appends the probability of the component being unavailable due to a failure at a specific time for a given number of hours ahead in time. A contingency enumeration approach, defining outage combinations as cutset structures [22,23] is used together with the time-series of expected unavailability of transmission lines to calculate annual Energy Not Supplied (ENS) for the system.

In this paper, a failure of a component is understood as a loss of ability to perform as required, leading to a fault, where the former is understood as an event, while the latter is understood as a state [24]. An outage is understood as the loss of ability of a component to deliver power, which may or may not cause an interruption of service to customers [25]. 
A contingency is understood as an outage occurrence of a single system component, or the concurrent outage of two or more system components [24]. A set is sometimes used interchangeably with a contingency. A cut-set is understood as a contingency which causes end-user consequences in terms of interrupted power. A cut-set is said to be minimal if the set cannot be reduced without loosing its status as a cut-set [26].

Protection systems are expected to be both dependable and secure [6,17]. Missing operation of the protection system occurs if it fails to react appropriately to a situation it is designed to respond to and would be a shortfall of the dependability of the system. Similarly would an unwanted operation occur if the protection system reacts to conditions it is not designed to react to, and is a shortfall of the security of the system [17]. This corresponds to the protection system misoperation types defined in [27]. Protection systems have previously been incorporated in power systems reliability analysis in multiple ways, such as through Markov models [14,16] and approximate methods [17,28].

Dependability and security were the basis in [17] to construct different scenarios in which protection system reliability can cause a transmission line to be isolated from the network. This gave rise to four fault types [17]: Fault type 1 (FT1) is the failure rate of the transmission line in focus. Fault type 2 (FT2) represents failures due to the spontaneous unwanted operation of a line's own protection system. Fault type 3 (FT3) is explained by a situation where a failure occurs on a neighboring line but is not correctly cleared due to missing operation of the neighboring line's protection system, and thus causes the line in focus to be isolated from the system. Fault Type 4 (FT4) is a result of a fault on the neighboring line that is correctly cleared by the neighboring line's protection system but causes an unwanted non-selective tripping of the line in focus. An equivalent failure rate for each line is then constructed as a summation of these four failure type contributions. The method is a contingency enumeration approach, where an approximate system reliability evaluation is used to obtain reliability indices for predefined minimal cut-sets. A more detailed presentation of the approach can be found in [17].

Complex network theory and graph-theoretic approaches have been used in the literature on cascading failures to study the power system, e.g., to identify critical components [29-31]. Protection system failures and misoperation have a propagating effect between components, and it is similarly necessary to consider the adjacency between them. The power system lends itself to a graph-based representation. A graph $(G)$ is an object consisting of an ordered vertex set $(V)$ and edge set $(E)$ joining the vertices through its two connected buses $u$ and $v$, as seen in (1)-(3), where $n$ is the number of vertices in the graph and $m$ is the number of edges in the graph, see e.g., [32,33]. Intuitively, it is natural to think of buses, $b$, as vertices and components such as transmission lines, $l$, as edges when it comes to representing the power system as a graph. An illustrative graph representation of a 4-bus power system following these conventions can be seen in Figure 1.

In the following, the graph-based representation of relationships between components is a useful tool to incorporate protection system failures into power system reliability analysis.

$$
\begin{gathered}
G=(V, E) \\
V=\left\{b_{1}, b_{2}, \ldots, b_{n}\right\} \\
E=\left\{l_{1}, l_{2}, \ldots, l_{m}\right\}=\left\{\left\{u_{1}, v_{1}\right\},\left\{u_{2}, v_{2}\right\}, \ldots,\left\{u_{m}, v_{m}\right\}\right\}
\end{gathered}
$$




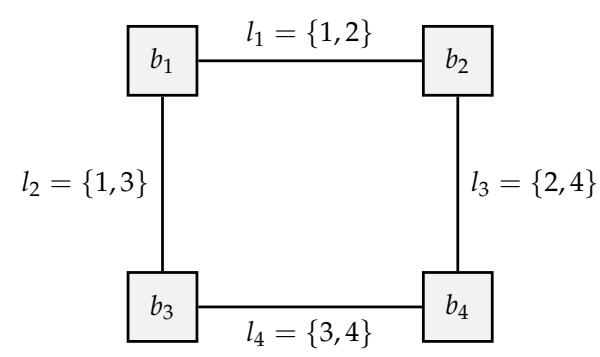

Figure 1. Illustrative graph of a 4-bus power system.

\section{Method}

In this section, a general and compact method for calculating reliability indices including protection system misoperations is postulated, using both approximate methods and a time-series method, based on the definitions of fault types as originally proposed in [17]. The basis for approximate methods is [34] in Section 3.1, and that for a time-series approach is [10] in Section 3.2.

In power systems, we normally look at buses as vertices and transmission lines as edges. However, the idea behind the proposed methodology in this paper is to see vertices as transmission lines and edges as dependencies. This structure can then be utilized to calculate the contribution to failure rates at a given transmission line, given failures at adjacent lines.

When including protection system failures into the system, we consider two different types of lines in each case: the target line $i$, for which we wish to calculate the failure rates, and the source line $j$, which is adjacent to the target line and contributes to the failure rate of the target line through propagation of protection system failures. The target- and source lines are adjacent lines if they are connected to a common bus, as seen in Figure 2. The subscript $l$ is used when it is not specific if the line is a target or a source line.

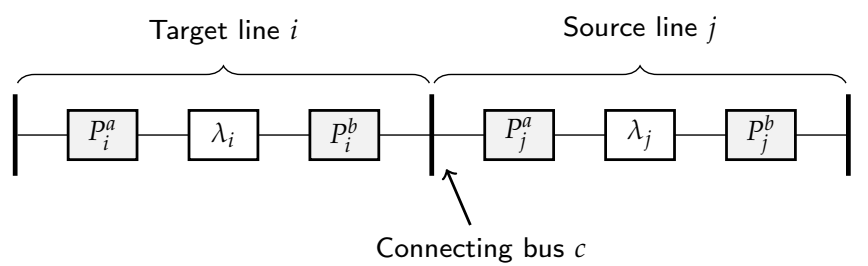

Figure 2. Two adjacent transmission lines, $i$ and $j$.

A line is associated with a protection system on each end. These are referred to as the a-side and the $b$-side protection system, represented as the set $s=\{a, b\}$ for simplicity. For a line $l$, the failure rate of the line $\lambda_{l}$, and the two protection systems, $P_{l}^{s}$ are considered. The protection systems have three parameters: (1) a specific annual failure rate, $\lambda_{l}^{s}$, (2) a conditional probability of missing operation, $p_{l}^{s, m}$, if the line experiences a failure, and (3) upon a correctly cleared failure of an adjacent line, a conditional probability of unwanted non-selective tripping at the target line, $p_{l}^{s, u}$. The associated outage duration, $r$, is denoted with a subscript indicating which line is considered, and a superscript indicating which fault type it is applicable to. All line- and protection system specific information is represented as ordered column vectors.

The primary concern is how misoperation of the protection system of one transmission line can cause an adjacent line to be isolated from the system. The system can initially be considered a graph $G$, where each edge $l=\{u, v\}$ represents a transmission line, and the buses are represented by vertices, which are unique observations of $u$ and $v$. Let $u$ represent the a-side connecting bus of a transmission line, and $v$ the b-side connecting bus of the line. An adjacency matrix indicates connections between vertices and is used to represent the system in a form where vertices are transmission lines and edges are directed paths of failure propagation between the lines. An adjacency matrix is constructed for 
each side of the source line, $\mathrm{A}^{\mathrm{s}}$ in (4), to take into account which side of the source line is connected to the target line. The adjacency matrix is an ordered $l * l$-matrix, where rows represent a target line $i$ and columns represent a source line $j$. If line $j$ is connected to line $i$ through its s-side, it is marked with a digit 1 in the appropriate element of the matrix, 0 otherwise. Matrices are typeset in uppercase regular font, vectors are typeset in lowercase bold italic, while scalar values such as specific elements of vectors or matrices are typeset with italic fonts.

$$
\begin{aligned}
& \mathrm{A}^{\mathrm{s}}=\left[a_{i, j}^{s}\right]=\left[\begin{array}{ccc}
a_{1,1} & \ldots & a_{1, l} \\
\vdots & \ddots & \vdots \\
a_{l, l} & \ldots & a_{l, l}
\end{array}\right], \text { where }: \\
& a_{i, j}^{a}= \begin{cases}1 & \text { if } u_{j} \in\left\{u_{i}, v_{i}\right\} \\
0 & \text { Otherwise }\end{cases} \\
& a_{i, j}^{b}= \begin{cases}1 & \text { if } v_{j} \in\left\{u_{i}, v_{i}\right\} \\
0 & \text { Otherwise }\end{cases} \\
& s \in\{a, b\} \\
& i \in\{1, \ldots, l\} \\
& j \in\{1, \ldots, l\}
\end{aligned}
$$

The system can now be represented as a transformed graph, where transmission lines are represented as vertices, and directed edges are possible paths of failure propagation due to protection system failures. Figure 3 gives a visual representation of the 4-bus system presented in Figure 1, where the adjacency matrices defines the directed edges between the source line and the target line in the graph.

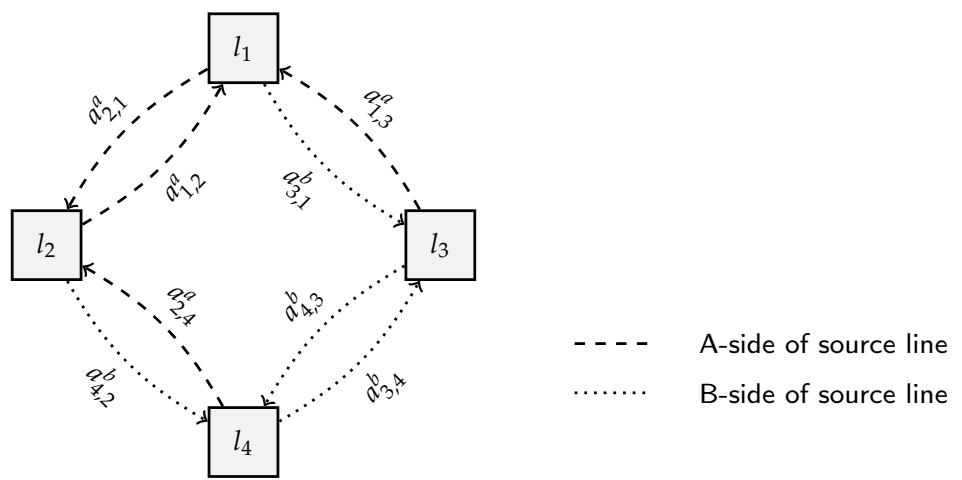

Figure 3. Transformed 4-bus system. Transmission lines as vertices, edges as propagation paths. Labeled edges correspond to elements in the resulting adjacency matrices marked with a digit 1.

The adjacency matrix can then be further modified to incorporate the probability that a failure on a source line will propagate to a target line. From the initial equations in [17], it is clear that only FT3 and FT4 stem from adjacent lines and as such these fault types will receive the primary focus in this paper.

FT3 is related to a failure on an adjacent line, which is not correctly cleared by the adjacent line's protection system. The probability of missing operation of the protection system on the $s$ side for a given line is represented by the column vector $p_{l}^{s, m}$. A matrix containing the probability that a failure propagates from the source line $j$ to the target line $i$ through the FT3 mechanism is created, named PT3, by modifying the adjacency matrix (5).

$$
\mathrm{PT} 3=\sum_{s}\left[\mathrm{~A}^{\mathrm{s}} \cdot \operatorname{diag}\left(p^{s, m}\right)\right]
$$


PT3 only takes into consideration the properties of line $j$ 's protection systems. The same probability matrix for FT4, a failure on the source line causing an unwanted nonselective tripping of the protection systems at the target line, $P T 4$, must consider protection system properties of both the target line and the source line. The probability of line $j$ successfully clearing a failure on its own line is included first in (6), followed by the probability of an unwanted non-selective tripping at line $i$, incorporated through transposing the matrix. Note that the probability of spontaneous unwanted operation is represented by a single column vector since the target line response is considered side-independent, where $p_{l}^{u}=P\left(p_{l}^{a, u} \cup p_{l}^{b, u}\right)$.

$$
\mathrm{PT} 4=\sum_{s}\left[\left[\mathrm{~A}^{\mathrm{s}} \cdot \operatorname{diag}\left(1-\boldsymbol{p}^{\mathrm{s}, \boldsymbol{m}}\right)\right]^{T} \cdot \operatorname{diag}\left(\boldsymbol{p}^{\boldsymbol{u}}\right)\right]^{T}
$$

Matrices representing the probability of different fault types propagating from a source line to a target line have now been established. These can be applied to calculate systems reliability indices for cut-sets. Two methods are presented here: an approximate method of system reliability using annual failure rates including protection system failures, and a method to calculate time series of probability of failure due to different fault types, which can be used together with [10] to calculate unavailability of cut-sets.

\subsection{Approximate Method}

This approach starts by calculating the equivalent failure rate of each line by considering each line a target line. The equivalent failure rate is calculated based on the failure rate of the line itself (FT1) and its protection systems (FT2), and the fault types FT3 and FT4 propagating from adjacent lines, following the method in [17]. FT1 and FT2 are only dependent on information of the line itself and is repeated for reference here (7) and (8). FT3 and FT4 are calculated by using the associated probability matrices (9) and (10). The multiplication of the matrices with the line failure rate vectors gives vectors of FT3 and FT4 failure rate contributions from all source lines at the target line.

$$
\begin{gathered}
f t 1_{i}=\lambda_{l} \\
f t 2_{i}=\sum_{s} \lambda_{l}^{s} \\
f t 3_{i}=\mathrm{PT} 3 \cdot \lambda_{l} \\
f t 4_{i}=\mathrm{PT} 4 \cdot \lambda_{l}
\end{gathered}
$$

Equivalent failure rate, incorporating protection system failures is then calculated as $\lambda^{\prime}$ in (11).

$$
\lambda^{\prime}=f t 1+f t 2+f t 3+f t 4
$$

From this, equivalent unavailability and outage durations for all target lines is found in (12) and (13), where the vectors of fault type contributions are paired with vectors of the associated repair or switching times using element-wise Hadamard operations (o signifies the Hadamard product, where $c_{i j}=a_{i j} \cdot b_{i j}$, and $\oslash$ is used for Hadamard division, see e.g., [35] for an introduction). A slight adjustment is made when incorporating unavailability due to FT3. As opposed to the other fault types, FT3 is dependent on the switching time at the source line, $j$, rather than that at the target line $i$. To include this, the contribution of the unavailability from the source line to target line is carried through the probability matrix for FT3.

$$
\begin{gathered}
U^{\prime}=f t 1 \circ r^{F T 1}+f t 2 \circ r^{F T 2}+\mathrm{PT} 3 \cdot\left[\lambda \circ r^{F T 3}\right]+f t 4 \circ r^{F T 4} \\
r^{\prime}=U^{\prime} \oslash \lambda^{\prime}
\end{gathered}
$$


Vectors containing reliability indices for single lines are now established. Second order cut-sets involving two lines $x$ and $y$ can now be calculated in a general form, avoiding a distinction in equations between adjacent and non-adjacent lines by utilizing the matrix of adjacency adjusted probabilities. If two lines in a cut-set are adjacent and they experience a FT3 or a FT4 where the source line is the other line in the cut-set, they will both surely be unavailable. This means that these dependent failures should be treated separately from the independent failures. Adjustments for dependent failures between individual lines in the cut-set are created in (14) and (15) before calculating the new expected restoration time of the cut-set due to independent failures in (16).

$$
\begin{gathered}
\lambda_{x}^{a}=\left(p t 3_{x, y}+p t 4_{x, y}\right) \cdot f t 1_{y} \\
U_{x}^{a}=\left(p t 3_{x, y} \cdot r_{y}^{F T 3}+p t 4_{x, y} \cdot r_{x}^{F T 4}\right) \cdot f t 1_{y} \\
r_{x}^{n}=\left(U_{x}^{\prime}-U_{x}^{a}\right) /\left(\lambda_{x}^{\prime}-\lambda_{x}^{a}\right)
\end{gathered}
$$

However, to account for both lines in the cut-set failing simultaneously due to the occurrence of a FT3 or FT4 of an adjacent line also in the cut-set, an added dependency mode failure rate, $\lambda^{D}$, is created in (17). The dependency mode failure rate and the expected unavailability due to these dependent events is used to calculate the associated expected restoration time in (18). It is important to note that if the lines are not adjacent, the elements $\{x, y\}$ and $\{y, x\}$ in the probability matrices will be zero, and hence all adjustments and the dependency mode failure rate will be zero. The failure rate of the cut-set will be calculated on the basis of independent component failures if the components are not adjacent.

$$
\begin{gathered}
\lambda_{\{x, y\}}^{D}=\lambda_{x}^{a}+\lambda_{y}^{a} \\
r_{\{x, y\}}^{D}= \begin{cases}\left(U_{x}^{a}+U_{y}^{a}\right) / \lambda_{\{x, y\}}^{D} & \text { if } \lambda_{\{x, y\}}^{D}>0 \\
0 & \text { Otherwise }\end{cases}
\end{gathered}
$$

The equivalent annual failure rate, expected annual unavailability and expected restoration time for the cut-set including any potential dependent failures are then calculated in (19)-(21).

$$
\begin{gathered}
\lambda_{\{x, y\}}^{\prime}=\frac{\left(\lambda_{x}^{\prime}-\lambda_{x}^{a}\right)\left(\lambda_{y}^{\prime}-\lambda_{y}^{a}\right)\left(r_{x}^{n}+r_{y}^{n}\right)}{8760}+\lambda_{\{x, y\}}^{D} \\
U_{\{x, y\}}^{\prime}=\frac{\left(\lambda_{x}^{\prime}-\lambda_{x}^{a}\right)\left(\lambda_{y}^{\prime}-\lambda_{y}^{a}\right)\left(r_{x}^{n} \cdot r_{y}^{n}\right)}{8760}+\lambda_{\{x, y\}}^{D} \cdot r_{\{x, y\}}^{D} \\
r_{\{x, y\}}^{\prime}=\frac{U_{\{x, y\}}^{\prime}}{\lambda_{\{x, y\}}^{\prime}}
\end{gathered}
$$

\subsection{Time Series Method}

Time series of failure probability can be used to incorporate varying failure probabilities due to exposure to external threats, e.g., wind, lightning, icing etc., leading to failure bunching effects. For time series, unavailability and associated reliability indices for cut-sets are calculated using the time series of different fault types, dependent on adjacency, as explained in this subsection. The algorithmic method outlined in [10] is used to calculate resulting unavailability and further reliability indices.

The addition of a time dimension to failure rates makes it necessary to make alterations to the equations in Section 3.1. The probability matrices established in Section 3 can still be used, assuming that the probability of a failure propagating is time independent. Timeseries of hourly failure probabilities for a set of lines, as presented in (22), is now considered. 
When referring to the time-series of failure probability for a single line, the column vector is denoted $\lambda_{:, 1}$. The fault types for a single target line $i$ are given in (23)-(27).

$$
\begin{gathered}
\lambda^{\mathrm{s}}=\left[\lambda_{t, l}^{s}\right]=\left[\begin{array}{ccc}
\lambda_{1,1} & \ldots & \lambda_{1, l} \\
\vdots & \ddots & \vdots \\
\lambda_{t, 1} & \ldots & \lambda_{t, l}
\end{array}\right] \\
\mathrm{FT}_{:, \mathrm{i}}=\lambda_{:, 1} \\
\mathrm{FT}_{:, \mathrm{i}}=\left[f t 2_{t, i}\right], \text { where } f t 2_{t, i}=\frac{\sum_{s} \lambda_{l}^{s}}{8760} \\
\mathrm{FT}_{:, \mathrm{i}}=\sum_{j}\left[\lambda_{:, j} \cdot p t 3_{i, j}\right] \\
\mathrm{FT}_{:, \mathrm{i}}=\sum_{j}\left[\lambda_{:, j} \cdot p t 4_{i, j}\right] \\
\lambda_{:, \mathrm{i}}^{\prime}=\mathrm{FT}_{:, \mathrm{i}}+\mathrm{FT} 2_{:, \mathrm{i}}+\mathrm{FT} 3_{:, \mathrm{i}}+\mathrm{FT} 4_{:, \mathrm{i}}
\end{gathered}
$$

When calculating the unavailability and failure probability of second order cut-sets it is necessary to adjust FT3 and FT4 to account for adjacency as was done in the approximate equations, for both lines $x$ and $y$, as seen in (28) and (29). A time series of dependency mode failure probability for the two lines in conjunction is calculated in (30). Again, the adjustments and dependency mode failure rate are equal to zero if the lines are not adjacent.

$$
\begin{gathered}
\mathrm{FT}_{:, \mathrm{x}}^{\prime}=\mathrm{FT} 3_{:, \mathrm{x}}-\mathrm{FT} 1_{:, \mathrm{y}} \cdot p t 3_{x, y} \\
\mathrm{FT}_{:, \mathrm{x}}^{\prime}=\mathrm{FT} 4_{:, \mathrm{x}}-\mathrm{FT} 1_{:, \mathrm{y}} \cdot p t 4_{x, y} \\
\lambda_{:,\{\mathrm{x}, \mathrm{y}\}}^{\mathrm{D}}=\mathrm{FT} 1_{:, \mathrm{y}} \cdot p t 3_{x, y}+\mathrm{FT} 1_{:, \mathrm{y}} \cdot p t 4_{x, y}+\mathrm{FT} 1_{:, \mathrm{x}} \cdot p t 3_{y, x}+\mathrm{FT} 1_{:, \mathrm{x}} \cdot p t 4_{y, x}
\end{gathered}
$$

For the cut-set itself, time-series of unavailability of individual lines with updated fault type values for FT3 and FT4 is calculated. The unavailability due to dependent faults is calculated as a separate time series, using restoration times according to the relevant line and the fault type. The calculation of unavailability is done using the method outlined in [10]. The result is a time series of the probability of the component being unavailable at a given point in time, which together with the unavailability due to the dependency mode failure probability is combined to the unavailability of the cut-set in (31). Once the time-series of unavailability is established, this is used to calculate the failure probability of the cut-set in (32).

$$
\begin{gathered}
\mathrm{U}_{:,\{x, y\}}^{\prime}=\mathrm{U}_{:, \mathrm{x}}^{\prime} \circ \mathrm{U}_{:, \mathrm{y}}^{\prime}+\mathrm{U}_{:,\{\mathrm{x}, \mathrm{y}\}}^{\mathrm{D}} \\
\lambda_{:,\{\mathrm{x}, \mathrm{y}\}}^{\prime}=\mathrm{U}_{:, \mathrm{x}}^{\prime} \circ \lambda_{:, \mathrm{y}}+\mathrm{U}_{:, \mathrm{y}} \circ \lambda_{:, \mathrm{x}}+\lambda_{:,\{\mathrm{x}, \mathrm{y}\}}^{\mathrm{D}}
\end{gathered}
$$

\section{Case Study}

A test case is constructed to exemplify the combined effect of failure bunching due to weather and protection system failures on power systems reliability. The test case is based on the topology and annual failure rates from the 24-bus IEEE Reliability Test System (RTS) [36] with added protection systems. Only Permanent Outage Rates (POR) for the branches are considered. A representation of the system with numbered branches can be seen in Figure 4. A contingency enumeration approach is employed to evaluate the reliability of the system. A consequence analysis is performed at peak load, using an AC OPF algorithm with load shedding, as described in [37] yielding interrupted power at load points for the different cut-sets. The calculated interrupted power is used together with the unavailability of the cut-sets to calculate their respective contributions to annual Expected Energy Not Supplied (ENS) of the system. 


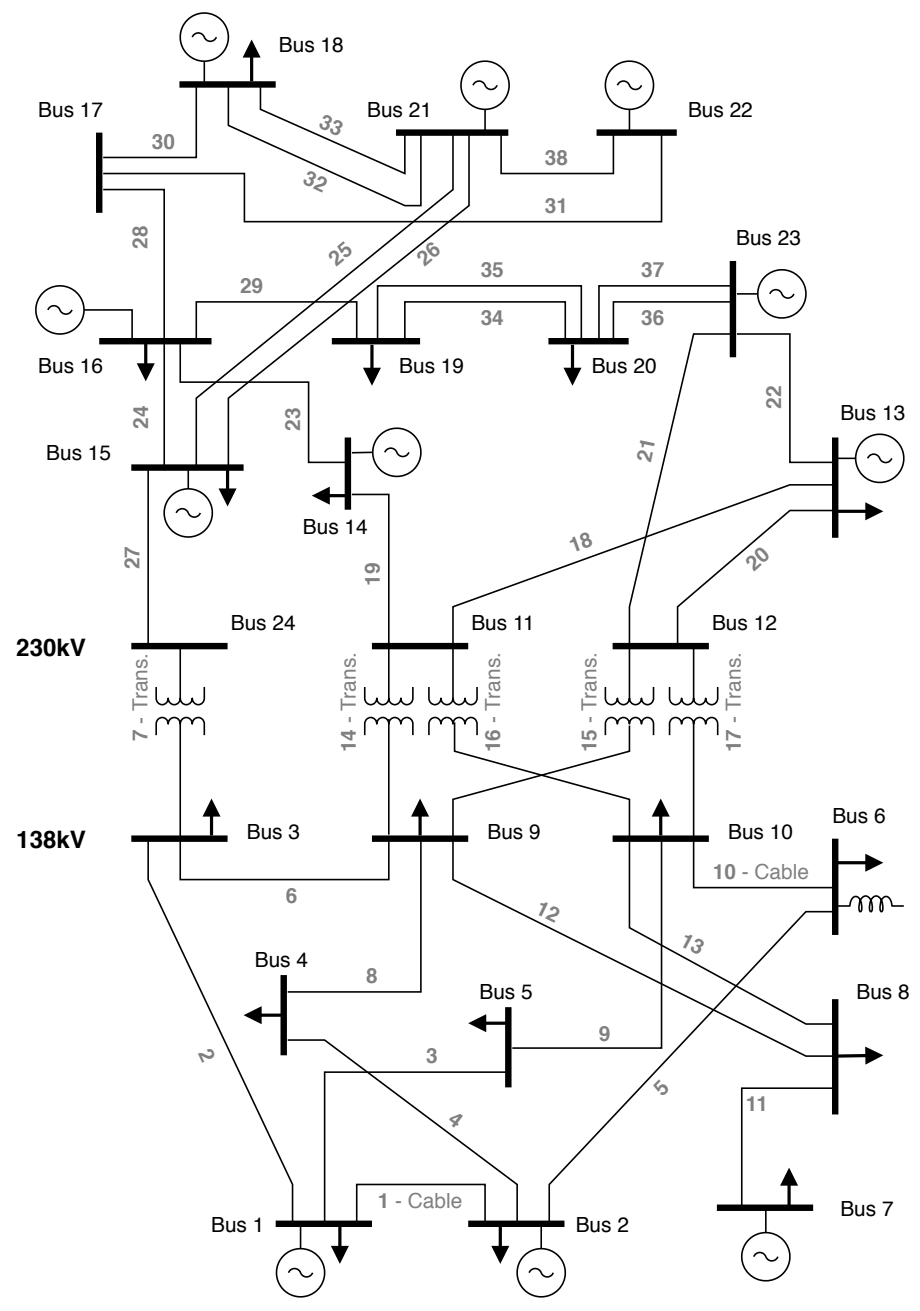

Figure 4. Twenty-four-bus Reliability Test System (RTS) [36] with component labels. Overhead transmission line unless otherwise noted.

Time series of hourly probability of failure due to wind conditions is calculated according to the method outlined in [19]. The time series covers 30 years of hourly estimated failure probabilities due to wind for actual lines in the Norwegian transmission system based on historical weather. This incorporates correlated weather exposure between lines in the data material. For the time-series, 75 percent of the failure probability is assumed to be constant, while 25 percent is scaled wind dependent failure probability varying at an hourly interval. Transformers and cables are assumed unaffected by wind, and are given a constant failure rate throughout the year. Annual failure rates due to permanent outages for the branches and the associated outage durations can be found in [36]. All protection systems are assumed to have an annual failure rate $\lambda_{l}^{s}=0.025$. The probability of missing operation of the protection system is assumed to be $P_{s}^{s, m}=0.0205$. The probability of unwanted non-selective tripping of the protection systems is assumed to be $P_{l}^{s, u}=0.007$. Repair of protection system units, relevant to FT2, is assumed to be $2 \mathrm{~h}$. FT3 and FT4 are associated with a $0.5 \mathrm{~h}$ switching time. These parameter values are in line with the case study presented in [17].

Unavailability of the different cut-sets are calculated in four ways: (1) A base case using an approximate method of reliability evaluation not including protection system failures [34]. (2) An approximate method including protection systems failures, as outlined in Section 3.1. (3) A time-series method including wind-dependent failure rates, as outlined in [10]. (4) A time-series method including wind-dependent failure rates adjusted for protection system faults, as outlined in Section 3.2. A simplification is done compared 
to the original approach in [10] when calculating time-series of unavailability, where restoration times are assumed to be constant values, rather than distributions.

Adjacency matrices are created and weighted according to dependent probabilities and failure rates following the procedure in Section 3 to incorporate protection system reliability into the analysis. The results can be illustrated for the single line, branch 35 , in Figure 5, where vertices are transmission lines and the directed edges show the adjacency between the lines. The line-style of the edges indicates the source line protection system side (A-side is dashed, B-side is dotted). To accumulate results, all edges leading into a vertex are summed up to see the contribution of FT3 and FT4 from the adjacent lines. Note the double connection between lines 34 and 35 appearing, causing line 34 to contribute to FT3 and FT4 at line 35 through two separate paths.
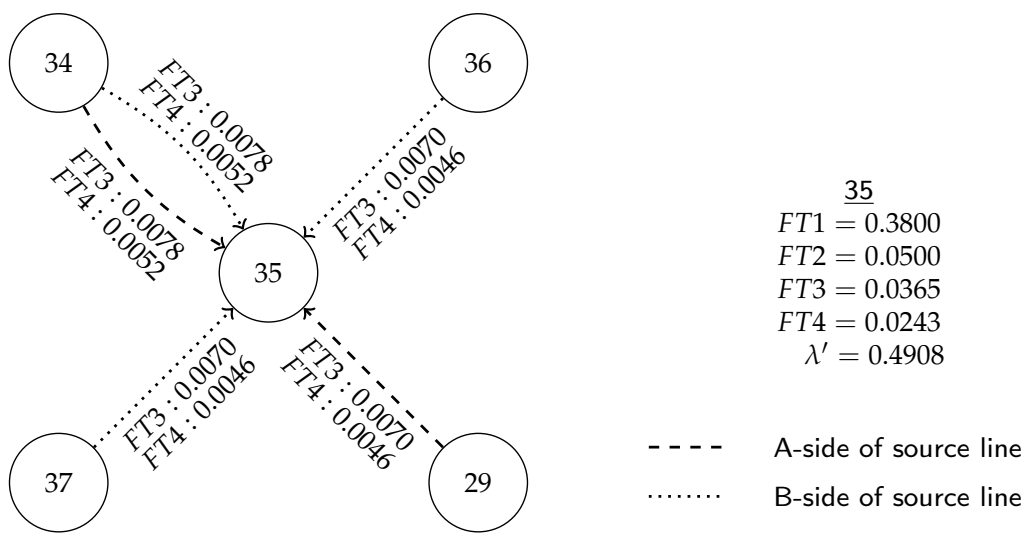

Figure 5. Contributions to fault type 3 (FT3) and FT4 for line 35 from adjacent lines. FT1-FT4 and adjusted failure rate for line 35 . Annual values.

Figure 6 shows a 40 hour sample of the probability of failure for the individual components in the cut-set $\{26,27\}$, as well as the calculated unavailability of the cut-set, to illustrate the method. The lines in the cut-set are adjacent at a single bus. In the top panel, the independent failure probabilities of the lines are relatively high, peaking around the tenth hour, while the dependency mode failure probability is comparably low. The dependency mode failure rate does however have a large impact on the failure probability of the cut-set due to its additive nature, as seen in the mid panel, where the failure probability of the cut-set is calculated with and without the inclusion of protection system failures. The resulting unavailability of the cut-set, illustrated in the lower panel, shows the impact of protection system failures as a short spike in unavailability compared to the case excluding protection system failures. This spike is due to the notably shorter outage durations associated with FT3 and FT4 of only thirty minutes, as compared with the permanent outage duration of independent faults for the lines at 11 hours. The unavailability only considering independent failures, with longer outage durations, is illustrated by the more rounded, dotted line.

The failure rates for sets of components in different categories are summarized in Table 1 . These are not necessarily cut-sets causing load curtailment for the RTS at peak load, however, but show the impact on failure rate and unavailability of the methods described in the paper on different sets of network components in different topologies. For single lines, the approximate and time-series methods yield similar results. This is expected, as the time-series method only points out when the failure occurs but does not alter the annual failure rate in any way. The inclusion of protection systems has a limited impact on the annual failure rate for individual cables and lines but a large impact for transformers. This is due to the initially low failure rate of the transformers, when paired with a comparably high failure rate of its protection systems affecting FT2 in addition to contributions from adjacent lines through FT3 and FT4. For second order sets where the components are not adjacent, the sets containing at least one component with a constant failure rate, e.g., cables, are similar across the methods. The inclusion of protection systems causes a small increase 
in the failure rate but there is no effect due to weather. However, when looking at sets including two overhead transmission lines, the effect of correlated weather causes a large increase in failure rate from the approximate method to the time series method, both not including protection systems. The failure rate increases sharply for sets where components are adjacent when protection systems are included in the evaluation. More importantly, the frequency of occurrence of some states is notably greater when protection systems and weather effects are both taken into consideration.
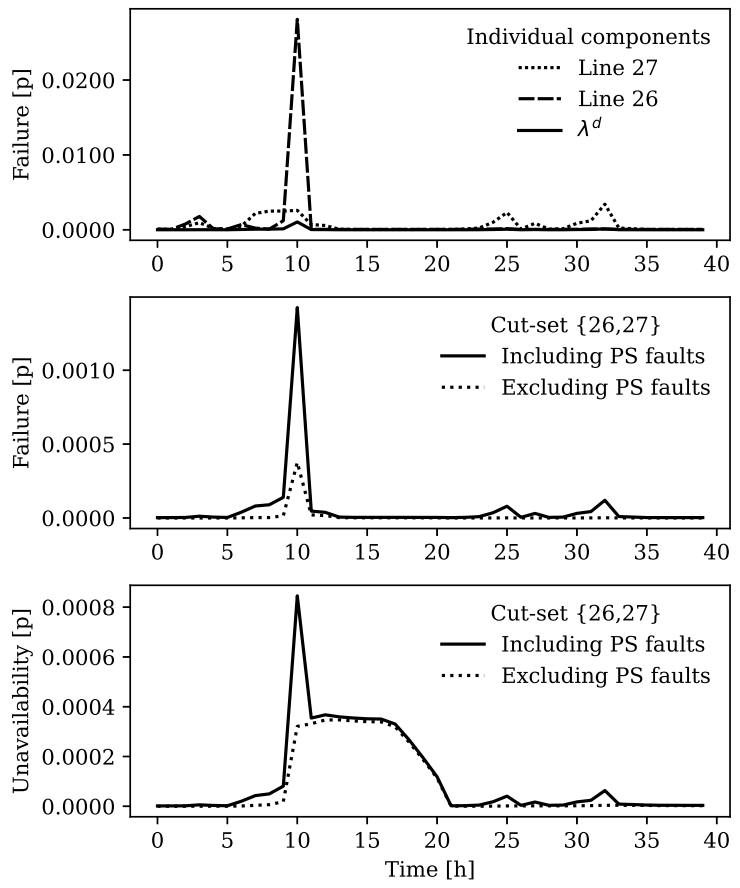

Figure 6. Individual failure probability of components in cut-set $\{26,27\}$ and dependency mode failure probability for a 40 hour time-window. Failure probability and unavailability of cut-set, including and excluding protection system failures.

Table 1. Annual failure rate for outages within different set categories $[\lambda / y]$.

\begin{tabular}{|c|c|c|c|c|c|}
\hline \multicolumn{2}{|c|}{ Set Information } & \multicolumn{4}{|c|}{ Method $^{1}$} \\
\hline \multirow[b]{2}{*}{ Connection } & \multirow[b]{2}{*}{ Components } & \multicolumn{2}{|c|}{ Approximate } & \multicolumn{2}{|c|}{ Time Series } \\
\hline & & No PS & PS & No PS & PS \\
\hline- & Cable & 0.570 & 0.773 & 0.570 & 0.773 \\
\hline- & Line & 12.250 & 15.534 & 12.250 & 15.534 \\
\hline- & Transformer & 0.100 & 0.673 & 0.100 & 0.673 \\
\hline None & Cable-Cable & 0.000 & 0.001 & 0.000 & 0.001 \\
\hline None & Cable-Line & 0.027 & 0.035 & 0.027 & 0.038 \\
\hline None & Cable-Trans & 0.004 & 0.006 & 0.004 & 0.006 \\
\hline None & Line-Line & 0.155 & 0.202 & 0.627 & 0.755 \\
\hline None & Line-Trans & 0.094 & 0.127 & 0.093 & 0.138 \\
\hline None & Trans-Trans & 0.000 & 0.003 & 0.000 & 0.003 \\
\hline 1 bus & Cable-Line & 0.003 & 0.172 & 0.003 & 0.173 \\
\hline 1 bus & Cable-Trans & 0.001 & 0.026 & 0.001 & 0.026 \\
\hline 1 bus & Line-Line & 0.020 & 1.477 & 0.111 & 1.581 \\
\hline 1 bus & Line-Trans & 0.015 & 0.330 & 0.015 & 0.332 \\
\hline 1 bus & Trans-Trans & 0.000 & 0.007 & 0.000 & 0.007 \\
\hline 2 buses & Line-Line & 0.001 & 0.204 & 0.010 & 0.214 \\
\hline
\end{tabular}

${ }^{1}$ Approximate and time-series method, excluding and including protection system failures (PS). 
In terms of unavailability of sets of components, Table 2 shows a somewhat different story. The single contingency of a transformer has a 5.7 times increase in failure probability when protection systems are included in the analysis but has nearly no change in expected unavailability. This is due to the short outage durations associated with protection systems failures in the model. Again, sets of components which are both exposed to correlated weather, such as overhead transmission lines, experience the largest increase in annual unavailability. The most extreme being the case of two overhead lines sharing both buses, where the time-series method including protection systems reliability has an expected unavailability 20 times higher than what is found using the approximate method without including protection systems failures.

ENS for the system calculated using the different methods is shown in Table 3. The transmission system is usually operated by the N-1 criterion, and only second order contingencies are considered here. Considering cut-sets consisting of two components with no shared buses, the majority of increase in ENS stems from the inclusion of weather effects in the model, affecting overhead transmission line cut-sets. For cut-sets with adjacent components, the inclusion of protection systems has the largest impact compared to the base case, although weather effects have almost the same impact as the inclusion of protection systems for overhead transmission line cut-sets within this category. The impact seen in second order cut-sets suggests that protection systems and weather effects are important aspects to consider when evaluating the reliability of supply.

Table 2. Annual unavailability for outages within different set categories $[h / y]$.

\begin{tabular}{|c|c|c|c|c|c|}
\hline \multicolumn{2}{|c|}{ Set Information } & \multicolumn{4}{|c|}{ Method } \\
\hline \multirow[b]{2}{*}{ Connection } & \multirow[b]{2}{*}{ Components } & \multicolumn{2}{|c|}{ Approximate } & \multicolumn{2}{|c|}{ Time Series } \\
\hline & & No PS & PS & No PS & PS \\
\hline- & Cable & 15.390 & 15.641 & 15.372 & 15.624 \\
\hline- & Line & 130.780 & 134.747 & 129.571 & 133.921 \\
\hline- & Transformer & 76.800 & 77.462 & 76.570 & 77.230 \\
\hline None & Cable-Cable & 0.005 & 0.005 & 0.005 & 0.005 \\
\hline None & Cable-Line & 0.206 & 0.215 & 0.203 & 0.212 \\
\hline None & Cable-Trans & 0.094 & 0.097 & 0.094 & 0.096 \\
\hline None & Line-Line & 0.826 & 0.876 & 3.415 & 3.575 \\
\hline None & Line-Trans & 0.988 & 1.027 & 0.976 & 1.009 \\
\hline None & Trans-Trans & 0.162 & 0.164 & 0.161 & 0.163 \\
\hline 1 bus & Cable-Line & 0.024 & 0.109 & 0.024 & 0.109 \\
\hline 1 bus & Cable-Trans & 0.041 & 0.053 & 0.040 & 0.053 \\
\hline 1 bus & Line-Line & 0.111 & 0.842 & 0.630 & 1.395 \\
\hline 1 bus & Line-Trans & 0.158 & 0.319 & 0.156 & 0.318 \\
\hline 1 bus & Trans-Trans & 0.108 & 0.112 & 0.107 & 0.111 \\
\hline 2 buses & Line-Line & 0.008 & 0.109 & 0.057 & 0.161 \\
\hline
\end{tabular}

Table 3. Energy Not Supplied (ENS) due to second order cut-sets, calculated using different methods; in MWh/year.

\begin{tabular}{cccccc}
\hline Cut-Set Information & \multicolumn{3}{c}{ Method } \\
& \multicolumn{2}{c}{ Approximate } & & \multicolumn{2}{c}{ Time Series } \\
\cline { 2 - 3 } \cline { 5 - 6 } Connection & No PS & PS & & No PS & PS \\
\hline Unconnected & 373 & 390 & & 999 & 1032 \\
of which is Line-Line & 211 & 222 & & 839 & 867 \\
Connected at 1 bus & 22 & 125 & & 107 & 214 \\
of which is Line-Line & 9 & 71 & & 94 & 160 \\
\hline Total & 395 & 515 & & 1107 & 1246 \\
\hline
\end{tabular}


The approach can support decision makers in taking appropriate actions based on risk and socioeconomic considerations. The revelation of cut-sets with a high ENS may guide prioritization in grid hardening efforts, such as enhancing robustness of lines, undergrounding of overhead transmission lines, or investment in new transmission routes. Identification of adjacent cut-sets with high failure rates due to propagating failures may similarly support prioritized inspection and maintenance efforts of the associated protection systems.

\section{Conclusions}

In this paper we have shown a compact and generalized method of including protection system failures in power system reliability analysis, based on a graph-theoretical approach. The use of adjacency matrices in the approach is novel. This incorporates the topology of the grid and possible paths of failure propagation due to protection system misoperation into the reliability analysis as a set of matrix operations. The method was extended further to account for time-series of failure probability in the analysis, allowing for inclusion of both protection system misoperation and time-varying failure probabilities throughout the year due to weather exposure.

Our investigations confirm the hypothesis that the combination of failure bunching effects and protection system failures adversely impact power system reliability. A case study was presented to show the effect on reliability of supply when weather exposure and protection system misoperation were implemented into the analysis. The case study shows that taking protection system reliability and the adjacency of transmission lines into account can have a large impact on the contribution to annual ENS from certain cut-sets due to the propagation of protection system failures. Since protection system misoperation follows an initial failure, propagating failures cluster around periods of high failure probability from other causes, and further increase risks associated with failure bunching effects. Thus, taking a time-series approach to capture time-varying failure rates including protection system failures can more accurately quantify the reliability of supply. This is especially important for more frequently occurring second order cut-sets. Taking the time-series approach would also be especially relevant when considering multiple operating states throughout the year. The results of the analysis can support decision makers in risk-based prioritization of grid hardening, inspection or maintenance efforts.

The compact and generalized method presented can be implemented with ease on large power systems. The results can be used to prioritize preventive and corrective measures aiming to reduce risks associated with unwanted events in the power system. The use of adjacency matrices to incorporate dependencies between component outages in the reliability evaluation was exemplified using an approximate method and an analytical timeseries method. The use of the probability matrices could, however, also be implemented into Monte Carlo based tools to the same effect.

Author Contributions: Conceptualization, E.S.K.; methodology, E.S.K.; original draft preparation, E.S.K.; review and editing, G.H.K.; visualization, E.S.K.; supervision, G.H.K. All authors have read and agreed to the published version of the manuscript.

Funding: The research leading to these results has received funding through the project "Analysis of extraordinary events in power systems" (HILP) (Grant No. 255226), co-funded by the Research Council of Norway, Statnett and Fingrid.

Institutional Review Board Statement: Not applicable.

Informed Consent Statement: Not applicable.

Data Availability Statement: Not applicable.

Conflicts of Interest: The authors declare no conflict of interest. The funders had no role in the design of the study; in the collection, analyses, or interpretation of data; in the writing of the manuscript, or in the decision to publish the results. 


\section{References}

1. Bompard, E.; Huang, T.; Wu, Y.; Cremenescu, M. Classification and trend analysis of threats origins to the security of power systems. Int. J. Electr. Power Energy Syst. 2013, 50, 50-64. [CrossRef]

2. Wang, Y.; Chen, C.; Wang, J.; Baldick, R. Research on Resilience of Power Systems under Natural Disasters-A Review. IEEE Trans. Power Syst. 2016, 31, 1604-1613. [CrossRef]

3. Panteli, M.; Mancarella, P. Influence of extreme weather and climate change on the resilience of power systems: Impacts and possible mitigation strategies. Electr. Power Syst. Res. 2015, 127, 259-270. [CrossRef]

4. Pourbeik, P.; Kundur, P.S.; Taylor, C.W. The anatomy of a power grid blackout. IEEE Power Energy Mag. 2006, 4, 22-29. [CrossRef]

5. Hines, P.; Apt, J.; Talukdar, S. Large blackouts in North America: Historical trends and policy implications. Energy Policy 2009, 37, 5249-5259. [CrossRef]

6. Papic, M.; Agarwal, S.; Allan, R.N.; Billinton, R.; Dent, C.J.; Ekisheva, S.; Gent, D.; Jiang, K.; Li, W.; Mitra, J.; et al. Research on Common-Mode and Dependent (CMD) Outage Events in Power Systems: A Review. IEEE Trans. Power Syst. 2017, 32, 1528-1536. [CrossRef]

7. Billinton, R.; Singh, G.; Acharya, J. Failure Bunching Phenomena in Electric Power Transmission Systems. Proc. Inst. Mech. Eng. Part O J. Risk Reliab. 2006, 220, 1-7. [CrossRef]

8. Billinton, R. Basic models and methodologies for common mode and dependent transmission outage events. In Proceedings of the 2012 IEEE Power and Energy Society General Meeting, San Diego, CA, USA, 22-26 July 2012; IEEE: Piscataway, NJ, USA, 2012. [CrossRef]

9. Panteli, M.; Mancarella, P. Modeling and evaluating the resilience of critical electrical power infrastructure to extreme weather events. IEEE Syst. J. 2017, 11, 1733-1742. [CrossRef]

10. Kiel, E.S.; Kjølle, G.H. Transmission line unavailability due to correlated threat exposure. In Proceedings of the 2019 IEEE Milan PowerTech, PowerTech 2019, Milano, Italy, 23-27 June 2019; IEEE: Milano, Italy, 2019. [CrossRef]

11. Guo, H.; Zheng, C.; Iu, H.H.C.; Fernando, T. A critical review of cascading failure analysis and modeling of power system. Renew. Sustain. Energy Rev. 2017, 80, 9-22. [CrossRef]

12. Dobson, I.; Newman, D.E. Cascading blackout overall structure and some implications for sampling and mitigation. Int. J. Electr. Power Energy Syst. 2017, 86, 29-32. [CrossRef]

13. Phadke, A.G.; Thorp, J.S. Expose hidden failures to prevent cascading outages. IEEE Comput. Appl. Power 1996, 9, 20-23. [CrossRef]

14. Billinton, R.; Tatla, J. Composite generation and transmission system adequacy evaluation including protection system failure modes. IEEE Trans. Power Appar. Syst. 1983, PAS-102, 1823-1830. [CrossRef]

15. Kaplan, S.; Garrick, B.J. On The Quantitative Definition of Risk. Risk Anal. 1981, 1, 11-27. [CrossRef]

16. Jiang, K.; Singh, C. New models and concepts for power system reliability evaluation including protection system failures. IEEE Trans. Power Syst. 2011, 26, 1845-1855. [CrossRef]

17. Vadlamudi, V.V.; Gjerde, O.; Kjølle, G.H. Dependability and security-based failure considerations in protection system reliability studies. In Proceedings of the 2013 4th IEEE/PES Innovative Smart Grid Technologies Europe, ISGT Europe 2013, Lyngby, Denmark, 6-9 October 2013; IEEE: Lyngby, Denmark, 2013. [CrossRef]

18. Kiel, E.S.; Kjølle, G.H. The impact of protection system failures and weather exposure on power system reliability. In Proceedings of the 2019 IEEE International Conference on Environment and Electrical Engineering and 2019 IEEE Industrial and Commercial Power Systems Europe (EEEIC/I\&CPS Europe), Genoa, Italy, 11-14 June 2019; IEEE: Piscataway, NJ, USA, 2019. [CrossRef]

19. Solheim, Ø.R.; Kjølle, G.H. Wind dependent failure rates for overhead transmission lines using reanalysis data and a Bayesian updating scheme. In Proceedings of the 2016 International Conference on Probabilistic Methods Applied to Power Systems (PMAPS), Beijing, China, 16-20 October 2016. [CrossRef]

20. Billinton, R.; Singh, G. Application of adverse and extreme adverse weather: Modelling in transmission and distribution system reliability evaluation. IEEE Proc. Gener. Transm. Distrib. 2006, 153, 115-120. [CrossRef]

21. Solheim, Ø.R.; Warland, L.; Trotscher, T. A holistic simulation tool for long-term probabilistic power system reliability analysis. In Proceedings of the 2018 IEEE International Conference on Probabilistic Methods Applied to Power Systems (PMAPS), Boise, ID, USA, 24-28 June 2018. [CrossRef]

22. Kjølle, G.H.; Gjerde, O. Integrated approach for security of electricity supply analysis. Int. J. Syst. Assur. Eng. Manag. 2010, 1, 163-169. [CrossRef]

23. Gjerde, O.; Kjølle, G.H.; Jakobsen, S.H.; Vadlamudi, V.V. Enhanced method for reliability of supply assessment-An integrated approach. In Proceedings of the 19th Power Systems Computation Conference, PSCC 2016, Genova, Italy, 20-24 June 2016; IEEE: Genoa, Italy, 2016. [CrossRef]

24. IEC 60050. Electropedia—International Electrotechnical Vocabulary; International Electrotechnical Commission: Geneva, Switzerland, 2009.

25. IEEE Standards Association. Proceedings of the 1366-2012-IEEE Guide for Electric Power Distribution Reliability Indices; IEEE Standards Association: Piscataway, NJ, USA, 2012. [CrossRef]

26. Rausand, M.; Høyland, A. System Reliability Theory: Models, Statistical Methods, and Applications, 2nd ed.; John Wiley \& Sons: New York, NY, USA, 2004.

27. Bian, J.J.; Slone, A.D.; Tatro, P.J. Protection system misoperation analysis. In Proceedings of the 2014 IEEE PES General Meeting I Conference \& Exposition, National Harbor, MD, USA, 27-31 July 2014; IEEE: Piscataway, NJ, USA, 2014; pp. 1-5. [CrossRef] 
28. Vadlamudi, V.V.; Gjerde, O.; Kjølle, G.H. Impact of protection system reliability on power system reliability: A new minimal cutset approach. In Proceedings of the 2014 International Conference on Probabilistic Methods Applied to Power Systems, PMAPS 2014, Durham, UK, 7-10 July 2014; IEEE: Durham, UK, 2014. [CrossRef]

29. Sun, K. Complex networks theory: A new method of research in power grid. In Proceedings of the IEEE Power Engineering Society Transmission and Distribution Conference, Dalian, China, 15-18 August 2005; IEEE: Piscataway, NJ, USA, 2005; pp. 1-6. [CrossRef]

30. Arianos, S.; Bompard, E.; Carbone, A.; Xue, F. Power grid vulnerability: A complex network approach. Chaos 2009, 19, 13119. [CrossRef] [PubMed]

31. Pagani, G.A.; Aiello, M. The Power Grid as a complex network: A survey. Phys. A Stat. Mech. Appl. 2013, 392, 2688-2700. [CrossRef]

32. Diestel, R. Graph Theory, 5th ed.; Graduate Texts in Mathematics; Springer: Berlin/Heidelberg, Germany, 2017; Volume 173, pp. xviii +428 . [CrossRef]

33. Chartrand, G.; Zhang, P. A First Course in Graph Theory—Chartrand, Zhang; Dover Publications: New York, NY, USA, $2012 ;$ p. 450.

34. Billinton, R.; Allan, R.N. Reliability Evaluation of Power Systems; Plenum Press: New York, NY, USA, 1996.

35. Styan, G.P. Hadamard products and multivariate statistical analysis. Linear Algebra Appl. 1973, 6, 217-240. [CrossRef]

36. IEEE Probability Methods Subcommittee. IEEE reliability test system. IEEE Trans. Power Appar. Syst. 1979, PAS-98, 2047-2054. [CrossRef]

37. Sperstad, I.B.; Jakobsen, S.H.; Gjerde, O. Modelling of corrective actions in power system reliability analysis. In Proceedings of the 2015 IEEE Eindhoven PowerTech, Eindhoven, The Netherlands, 29 June-2 July 2015; IEEE: Piscataway, NJ, USA, 2015. [CrossRef] 